\title{
On vorticity waves propagating in a waveguide formed by two critical layers
}

\author{
OLEG DERZHO ${ }^{1,2}$ AND ROGER GRIMSHA W ${ }^{3} \dagger$ \\ ${ }^{1}$ Department of Physics and Physical Oceanography, Memorial University of Newfoundland, \\ St. John's, NL A1B 3X7, Canada \\ ${ }^{2}$ Institute of Thermophysics, Russian Academy of Sciences, Novosibirsk 630090, Russia \\ ${ }^{3}$ Department of Mathematical Sciences, Loughborough University, Leics LE11 3TU, UK
}

(Received 21 October 2008 and in revised form 15 January 2009)

A theoretical model for long vorticity waves propagating on a background shear flow is developed. The basic flow is assumed to be confined between two critical layers, respectively, located near the lower and upper rigid boundaries. In these critical layers even small disturbances will break, and eventually a thin zone of mixed fluid will appear. We derive a nonlinear evolution equation for the amplitude of a wave-like disturbance in this configuration, based on the assumption that the critical layers are replaced by thin recirculation zones attached to the lower and upper rigid boundaries, where the flow is very weak. The dispersive and time-evolution terms in this equation are typical for Korteweg-de Vries theory, but the nonlinear term is more complicated. It comprises nonlinearity associated with the shear across the waveguide, and the nonlinearity due to the flow over the recirculation zones. The coefficient of the quadratic nonlinear term may change sign, depending on the presence or otherwise of recirculation zones at the upper or lower boundary of the waveguide. We then seek steady travelling wave solutions, and show that there are no such steady solutions if the waveguide contains no density stratification. However, steady solutions including solitary waves and bores can exist if the fluid between the critical layers is weakly density stratified.

\section{Introduction}

Solitary waves of permanent form propagating in a density-stratified shear flow owe their existence to a balance between nonlinear wave steepening and linear wave dispersion. For waves of small but finite amplitude the nonlinearity is usually quadratic and the governing equation is then the well-known Korteweg-de Vries (KdV) equation (see Benney 1966; Maslowe \& Redekopp 1980; Grimshaw 2001 for instance). Higherorder expansions in the wave amplitude may account for stronger nonlinearity, but such an approach is limited to waves that do not overturn. Overturning occurs at certain finite wave amplitudes for which the horizontal velocity approaches zero in a frame of reference moving with the wave, that is, a local critical point appears inside the flow. Above this critical amplitude a recirculation zone (or vortex core) may be generated, which then crucially alters the nature of the wave. In our previous works (Derzho \& Grimshaw 1997, 2007) we have found certain situations in which the recirculation zone gives rise to an extra nonlinearity proportional to the $3 / 2$ power 
of the difference between the wave amplitude and the critical amplitude, where the form of this nonlinear term does not depend on the details of the stratification and shear. However, in these previous works we assumed that the ambient stratification and shear were nearly uniform, with the consequence that although overturning occurred at some finite amplitude, there were no critical layers at the leading order.

On the contrary, in this study we suppose instead that the background shear flow is essentially non-uniform and bounded by two critical layers which are adjacent to the rigid walls. In this case even a small amplitude disturbance will overturn, with consequent mixing and the formation of a recirculation zone. Another important difference compared to our previous study of internal waves is that the waves considered here may exist even in a homogeneous fluid, although we will show that some weak stratification is needed to allow for the existence of steady waves. In this paper the waves owe their existence to the gradient of vorticity arising due to the ambient shear flow and thus may be called vorticity waves. This is in marked contrast with the usual internal wave situation (Maslowe \& Redekopp 1980; Grimshaw 2001) where the shear in effect only modifies the role of the density stratification. Such vorticity waves have been intensively studied, for instance in the context of plane Poiseuille flow in a channel (Pedley \& Stephanoff 1985) and in the dynamics of the upper ocean (Shrira 1989). The latter paper gave rise to several geophysical studies, but the presence of a thin shear layer near one boundary of the waveguide was an essential physical feature. A typical difficulty associated with this class of problems is a proper resolution of the flow near and inside the critical layer. In general, this would involve a matching between the possibly diverging interior solutions and the critical layer dynamics (see the review by Maslowe 1986 for instance). We note, however, that for certain two-dimensional boundary layer flows, the leading-order dynamics is not affected by the detailed critical layer dynamics (see Kachanov, Ryzhov \& Smith 1993 for instance). A similar conclusion was reached by Shrira (1989) and Voronovich, Shrira \& Stepanyants (1998) for certain classes of free surface flows.

Our present paper deals with waves in the waveguide formed by two critical layers, one at each rigid channel boundary. We assume that in either or both of these critical layers, the waves overturn and mixing takes place. Then after a long time, a recirculation zone of constant density forms in which it transpires that the flow is quite weak. It turns out that there is then no need to match the diverging part of the interior solution with a critical layer expansion. Instead, we follow the procedure of our previous studies (Derzho \& Grimshaw 1997, 2007) and present a technique that explicitly resolves the structure of these recirculation zones. Then we match this solution across the boundary of the recirculation zone, this boundary also being an unknown variable, with the interior solution. The outcome is a nonlinear evolution equation describing long-wave disturbances of small amplitude propagating along this waveguide. As might be expected, the dispersive and time-evolution terms in this equation are the same as in the usual KdV theory, but the nonlinear terms are much more complicated. Essentially the nonlinearity is both due to the nonlinearity associated with the shear flow across the waveguide, and the nonlinearity due to the flow over the recirculation zones of mixed fluid. We will show that the sign of the coefficients of the resulting quadratic nonlinear terms depends on whether the recirculation zone is located near the upper or the lower boundary of the waveguide. Further, the nonlinearity due to the flow over the recirculation zones may effectively cancel the nonlinearity associated with the ambient shear flow. We will examine steady travelling wave solutions of these evolution equations, and show that there 
are no steady vorticity waves in this geometry if the fluid has a constant density. However, a weak density stratification adds a linear term to the evolution equation, which although can it be safely neglected at the leading order, in fact controls the existence of nonlinear steady travelling vorticity waves in this situation. Then we describe in detail the possible steady solitary wave solutions, which include internal bores. All these solutions essentially differ from typical vorticity waves. The paper is organized as follows, in $\S 2$, we formulate the unsteady equations of motion, identify all relevant small parameters and describe the asymptotic construction of vorticity waves propagating over recirculation zones of mixed fluid. In $\S 3$, we discuss the possible steady travelling waves. Then, in $\S 4$, we present a brief conclusion.

\section{Formulation}

We consider the usual equations for the two-dimensional flow of an ideal densitystratified fluid with an ambient shear current $U_{0}(z)$ in the Boussinesq approximation. The flow is confined in a channel of depth $H$, and the maximum of the ambient flow velocity is $U_{m}$. Thus in dimensionless form the governing equations are

$$
\left(\nabla^{2} \psi\right)_{t}+J\left(\nabla^{2} \psi, \psi\right)+\frac{g H}{U_{m}^{2}} \rho_{x}=0, \quad \rho_{t}+J(\rho, \psi)=0,
$$

where $J(a, b)=a_{x} b_{z}-a_{z} b_{x}$ and $\nabla^{2}$ is the Laplacian operator. Here $\psi$ is the streamfunction such that the respective velocity fields are given by $u=\psi_{z}$ and $v=-\psi_{x}$. The dimensionless streamfunction is scaled with $U_{m} H$, space and time are scaled with $H$ and $H / U_{m}$, respectively, while the density $\rho$ is scaled with an appropriate constant $\rho_{00}$. The boundary conditions are that

$$
\psi_{x}=0 \quad \text { at } \quad z=0,1 .
$$

We consider long (compared to the waveguide depth $H$ ) waves, travelling with speed $c$ and undergoing a slow evolution with time. Hence we introduce slow space and time variables as follows:

$$
X=\mu(x-c t), \quad \tau=\mu^{3} t
$$

where $\mu \ll 1$ is a long-wave aspect ratio. In this study our focus is on vorticity waves which owe their primary existence to the gradient of the shear flow. That is, waves where gravity is the principal restoring force are excluded from consideration. Hence, we seek solutions of (2.1) in the form of asymptotic expansions in powers of $\mu$,

$$
\begin{aligned}
& \psi=\psi^{(0)}\left(z, \mu^{2}\right)+\mu^{2} \psi^{(1)}(X, z, \tau)+\mu^{4} \psi^{(2)}(X, z, \tau)+\cdots, \\
& \rho=1+\sigma\left(\rho^{(0)}(z)+\mu^{2} \rho^{(1)}(X, z, \tau)+\cdots\right) .
\end{aligned}
$$

Here $\sigma=\Delta \rho / \rho_{00} \ll 1, \Delta \rho=\rho_{-}-\rho_{+}$, where $\rho_{-}$and $\rho_{+}$are the basic state densities at the lower and upper boundaries of the waveguide, respectively. We suppose that the stratification is weak so that

$$
\lambda=\frac{\sigma g H}{U_{m}^{2}} \sim \mu^{2} \ll 1 .
$$

Note that otherwise the density stratification is arbitrary.

Then the zeroth-order approximation (the terms $\sim \mu^{2}$ in (2.1)) yields the ambient shear current

$$
\psi^{(0)}\left(z, \mu^{2}\right)=\int_{0}^{z} U\left(z, \mu^{2}\right) \mathrm{d} z
$$


Note that the ambient shear current $U\left(z, \mu^{2}\right)$ also depends on $\mu^{2}$ so that

$$
U\left(z, \mu^{2}\right)=U_{0}(z)+\mu^{2} U_{1}(z)+\cdots .
$$

A similar expansion could be imposed for the basic density field, but is not needed here. Then, in order to create critical layers at the boundaries, we will suppose that

$$
U_{0}(0)=U_{0}(1)=c, \quad U_{1}(0)=\Delta_{-}, \quad U_{1}(1)=\Delta_{+},
$$

where $\Delta_{ \pm}$denotes a detuning at the upper $(z=1)$ and lower $(z=0)$ boundaries, respectively.

The usual approach for solving problem (2.1) and (2.2) requires finding a set of eigenmodes satisfying the boundary value problem, with $c$ as an eigenvalue, which is to be determined. But for the problem considered here, $c$ is fixed and at the leading order is equal to the magnitude of the ambient shear flow at the boundaries. That is, here the waveguide is created by a pair of critical layers. Formally, it is readily shown that the first-order terms ( $\sim \mu^{3}$ terms in (2.1) satisfying the boundary conditions (2.2)) are

$$
\psi^{(1)}(z, X, \tau)=A(X, \tau)\left(U_{0}(z)-c\right),
$$

From (2.1) it is readily found that, again to the leading order,

$$
\rho^{(1)}(z, X, \tau)=A(X, \tau) \rho_{z}^{(0)}
$$

The second-order approximation leads to the following equation:

$$
\begin{gathered}
\psi_{z z}^{(2)}\left(U_{0}-c\right)-\psi^{(2)} U_{0 z z}=F^{(2)} \\
\text { where } F_{X}^{(2)}=\frac{\lambda}{\mu^{2}} A_{X} \rho_{z}^{(0)}-A_{X}\left(U_{1} U_{0 z z}-\left(U_{0}-c\right) U_{1 z z}\right)-A_{\tau} U_{0 z z} \\
-A_{X X X}\left(U_{0}-c\right)^{2}-A A_{X}\left\{U_{0 z z} U_{0 z}-\left(U_{0}-c\right) U_{0 z z z}\right\} .
\end{gathered}
$$

The requirement that all terms in (2.13) are of the same order leads to (see (2.6))

$$
\lambda \sim \mu^{2}
$$

as well as to the justification of the time scaling in (2.3).

Before proceeding to solve this equation for $\psi_{X}^{(2)}$ we note that there are critical layers at the boundaries $z=0,1$ and these must be resolved separately. This is carried out in detail below, but for now we note that the critical layers are resolved by thin recirculation zones $0<z<\eta_{-}(X, \tau)$ and $\eta_{+}(X, \tau)<z<1$, and hence we consider (2.12) in the region $\eta_{-}<z<\eta_{+}$. Next we note that there is a particular solution corresponding to the terms in $U_{1}$ in $F^{(2)}$, so that we may write

$$
\psi^{(2)}=\tilde{\psi^{(2)}}+U_{1} A \text {. }
$$

Integration over the channel then yields

$$
\left[U_{0 z} \tilde{\psi}_{X}^{(2)}\right]_{\eta_{-}}^{\eta_{+}}=\frac{\lambda}{\mu^{2}} A_{X}+A_{\tau}\left[U_{0 z}\right]_{\eta_{-}}^{\eta_{+}}+A_{X X X} \int_{\eta_{-}}^{\eta_{+}}\left(U_{0}-c\right)^{2} \mathrm{~d} z+A A_{X}\left[U_{0 z}^{2}\right]_{\eta_{-}}^{\eta_{+}},
$$

where $\rho^{(0)}\left(\eta_{-}\right)-\rho^{(0)}\left(\eta_{+}\right)=1$, which follows from the definition of $\sigma$. Note that $\sigma \geqslant 0$ and $\lambda \geqslant 0$ for stable stratification. In obtaining this equation we have used the result that the leading order $U_{0}-c$ vanishes in the critical layers, as discussed below. In order to obtain the amplitude $A(X, \tau)$ from (2.16) we need to find $\widetilde{\psi}_{X}^{(2)}$ at the critical layer boundaries, as well as $\eta_{+}$and $\eta_{-}$. When there is no wave motion $A=0$, and so $\eta_{+}=1$ and $\eta_{-}=0$. 
To satisfy the stability criterion in the lowest order we shall assume that $U_{0}(z)$ has no inflection points in the interior between the two boundaries. Further, critical layer condition (2.9) implies that $U_{0 z}(0)$ and $U_{0 z}(1)$ have opposite signs. We will consider a jet-like profile (a wake-like profile can be treated analogously) so that

$$
U_{0 z}(0)>0>U_{0 z}(1)
$$

Our key observation is that the horizontal velocity (in the frame of reference moving with the wave) may change sign, and this change initially occurs near either the lower or upper boundary, depending on the sign of $A$. Then, our main physical assumption is that where reversed flow is predicted, that is where $\psi_{z}-c \leqslant 0$, then mixing occurs in that region. This leads to the trapping of mixed fluid in a recirculation zone which exists between the wall and a free boundary defined by the critical layer condition $\psi_{z}=c$. As discussed in our previous works (Derzho \& Grimshaw 1997, 2007) the thin depth $\delta$ of the recirculation zone relative to its along-channel length implies that the velocity field there (in the reference frame moving with the wave) must be quite small, specifically $O\left(\delta^{2}\right)$. Effectively, the flow is stagnant in the frame of reference moving with the wave. Thus, we define the recirculation zone boundaries where $\psi_{z}=c$, by

$$
\eta_{+}=1-\delta f_{+} \quad \text { and } \quad \eta_{-}=0+\delta f_{-} .
$$

It follows that

$$
\begin{gathered}
\psi_{z}-c=\mu^{2}\left(A(X, \tau) U_{0 z}(0)+\Delta_{-}\right)+\delta U_{0 z}(0) f_{-}+\cdots=0 \quad \text { at } z=\eta_{-} \\
\psi_{z}-c=\mu^{2}\left(A(X, \tau) U_{0 z}(1)+\Delta_{+}\right)-\delta U_{0 z}(1) f_{+}+\cdots=0 \quad \text { at } z=\eta_{+} \\
\text {and so } \delta f_{-}=-\mu^{2} \Delta_{-} / U_{0 z}(0)-\mu^{2} A(X, \tau), \\
\text { while } \delta f_{+}=\mu^{2} \Delta_{+} / U_{0 z}(1)+\mu^{2} A(X, \tau) .
\end{gathered}
$$

The requirement that all terms in (2.21) and (2.22) are of the same order leads to the scaling

$$
\delta=\mu^{2}
$$

It is now important to note that $f_{-} \geqslant 0$ and $f_{+} \geqslant 0$ as negative values of $f_{-}$and $f_{+}$are prohibited since there are rigid boundaries at $z=0,1$ that confine the flow. Therefore

$$
\begin{aligned}
f_{-}>0 \text { and } f_{+}>0 & \text { if } \quad A_{* 1}<A<A_{* 0}, \\
f_{-}=0 & \text { if } A \geqslant A_{* 0}, \\
f_{+}=0 & \text { if } A \leqslant A_{* 1}, \\
\text { where } A_{* 1}=-\Delta_{+} / U_{0 z}(1) & \text { and } A_{* 0}=-\Delta_{-} / U_{0 z}(0) .
\end{aligned}
$$

When $A \geqslant A_{* 0}$ (2.21) gives the formula for $f_{-}$and similarly (2.22) does so for $f_{+}$if $A \leqslant A_{* 1}$. There are two distinct cases, namely, $A_{* 0}>A_{* 1}$ when $\Delta_{-}<\Delta_{+} U_{0 z}(0) / U_{0 z}(1)$ and $A_{* 0} \leqslant A_{* 1}$ that corresponds to $\Delta_{-}>\Delta_{+} U_{0 z}(0) / U_{0 z}(1)$. The former case will be considered first.

Since the recirculation zones are stagnant to leading order, and the density is continuous across the boundary of each nonlinear critical layer, the kinematic boundary condition, which is in full,

$$
\psi_{X}=-\mu^{2} \eta_{\tau}-\left(\psi_{z}-c\right) \eta_{X} \quad \text { at } \quad z=\eta
$$


can be applied at each boundary $z=\eta_{ \pm}$to yield a relation between $\tilde{\psi}^{(2)}$ and $f_{ \pm}$ and $A$,

$$
\begin{aligned}
& \left\{\begin{array}{l}
\tilde{\psi}_{X}^{(2)}(0)=A_{\tau}+A A_{X} U_{0 z}(0) \\
\tilde{\psi}_{X}^{(2)}(1)=-A_{X} \Delta_{+}
\end{array}\right\} \quad \text { if } \quad A \leqslant A_{* 1}, \\
& \left\{\begin{array}{c}
\tilde{\psi}_{X}^{(2)}(0)=A_{\tau}+A A_{X} U_{0 z}(0) \\
\tilde{\psi}_{X}^{(2)}(1)=A_{\tau}+A A_{X} U_{0 z}(1)
\end{array}\right\} \quad \text { if } \quad A_{* 1}<A<A_{* 0}, \\
& \left\{\begin{array}{l}
\tilde{\psi}_{X}^{(2)}(0)=-A_{X} \Delta_{-} \\
\tilde{\psi}_{X}^{(2)}(1)=A_{\tau}+A A_{X} U_{0 z}(1)
\end{array}\right\} \quad \text { if } \quad A \geqslant A_{* 0} .
\end{aligned}
$$

Finally the evolution equation for the wave amplitude is as follows:

$$
\begin{gathered}
\frac{\lambda}{\mu^{2}} A_{X}+\gamma A_{X X X}+F_{-}(A)=0, \\
F_{-}(A)=\left\{\begin{array}{ll}
A A_{X} U_{0 z}^{2}(1)+\Delta_{+} U_{0 z}(1) A_{X}+A_{\tau} U_{0 z}(1), & \text { if } A \leqslant A_{* 1}, \\
0, & \text { if } A_{* 1}<A<A_{* 0}, \\
-A A_{X} U_{0 z}^{2}(0)-\Delta_{-} U_{0 z}(0) A_{X}-A_{\tau} U_{0 z}(0), & \text { if } A \geqslant A_{* 0},
\end{array}\right\} \\
\text { and } \gamma=\int_{0}^{1}\left(U_{0}-c\right)^{2} \mathrm{~d} z>0 .
\end{gathered}
$$

Next let us consider the case $\Delta_{-}>\Delta_{+} U_{0 z}(0) / U_{0 z}(1)$ so that $A_{* 0}<A_{* 1}$ and then both $f_{-}=0$ and $f_{+}=0$ (i.e. there are no recirculation zones near the boundaries) within the interval $A_{* 0}<A<A_{* 1}$. A procedure similar to that presented above leads to the evolution equation

$$
\begin{gathered}
\frac{\lambda}{\mu^{2}} A_{X}+\gamma A_{X X X}+F_{+}(A)=0, \\
F_{+}(A)=\left\{\begin{array}{ll}
A A_{X} U_{0 z}^{2}(1)+\Delta_{+} U_{0 z}(1) A_{X}+A_{\tau} U_{0 z}(1), & \text { if } A \leqslant A_{* 0} \\
A A_{X}\left(U_{0 z}^{2}(1)-U_{0 z}^{2}(0)\right)+A_{\tau}\left(U_{0 z}(1)-U_{0 z}(0)\right) & \text { if } A_{* 0}<A<A_{* 1} \\
+A_{X}\left(\Delta_{+} U_{0 z}(1)-\Delta_{-} U_{0 z}(0)\right), & \text { if } A \geqslant A_{* 1} \\
-A A_{X} U_{0 z}^{2}(0)-\Delta_{-} U_{0 z}(0) A_{X}-A_{\tau} U_{0 z}(0), &
\end{array}\right\} .
\end{gathered}
$$

When $A_{* 0}<A<A_{* 1}$ there is no nonlinear term associated with the recirculation zones as these are absent in this amplitude range, so (2.35) then has the form typical for vorticity waves without trapped mixing zones.

The final equations (2.32) and (2.35) with the appropriate choice of $F_{-}$or $F_{+}$ depending on (the sign of) $\Delta_{-}-\Delta_{+} U_{0 z}(0) / U_{0 z}(1)>0$ or $<0$ is the principal result of our study. Solutions of these equations will be considered in the next section.

\section{Solutions of the amplitude evolution equation}

Extensive examination of the solutions of the final evolution equation requires numerical simulations, and is beyond the scope of the present paper. However, steady travelling wave solutions are quite simple to study using analytical methods. We put $A_{\tau}=0$ as we have already fixed the relation between wave celerity and the ambient 
flow (2.9) (otherwise only a re-determination of $\Delta_{ \pm}$is needed). We integrate (2.32) and (2.35) once to get the steady form of the resulting equation.

Consider first the case $\Delta_{-}<\Delta_{+} U_{0 z}(0) / U_{0 z}(1)$ when

$$
\begin{gathered}
\gamma A_{X X}=-\Pi_{A}(A) \\
\Pi_{A}(A)=\frac{\lambda}{\mu^{2}} A+\left\{\begin{array}{lll}
U_{0 z}^{2}(1)\left(A-A_{* 1}\right)^{2} / 2+\alpha_{0}, & \text { if } \quad A \leqslant A_{* 1} \\
\alpha_{0}, & \text { if } \quad A_{* 1}<A<A_{* 0} \\
-U_{0 z}^{2}(0)\left(A-A_{* 0}\right)^{2} / 2+\alpha_{0}, & \text { if } A \geqslant A_{* 0}
\end{array}\right\} .
\end{gathered}
$$

Here $\alpha_{0}$ is a constant of integration, and the other constants in (3.2) are determined to ensure the continuity of $A_{X X}$. A qualitative analysis of the solutions of (3.2) can then be done using the mechanical analogy with a particle in a potential well, where the potential energy of the particle is denoted by $\Pi$. First we examine the case of a homogeneous layer, that is $\lambda=0$. It can then be shown that there is only one extremum of $\Pi$ which means that there is no steady solution for any shear flow profile. This situation persists for small $\lambda>0$; but for sufficiently large $\lambda>0$ three extreme points emerge, and so there can then exist a family of periodic waves, which includes a solitary wave as a limiting case.

Next we turn to the alternative case $\Delta_{-}>\Delta_{+} U_{0 z}(0) / U_{0 z}(1)$ when $A_{* 0}<A_{* 1}$, so that

$$
\Pi_{A}(A)=\frac{\lambda}{\mu^{2}} A+\left\{\begin{array}{ccc}
U_{0 z}^{2}(1)\left(A-A_{* 1}\right)^{2} / 2+\alpha_{0}, & \text { if } & A \leqslant A_{* 0} \\
U_{0 z}^{2}(1)\left(A-A_{* 1}\right)^{2} / 2-U_{0 z}^{2}(0) & & \\
\times(\cdots)\left(A-A_{* 0}\right)^{2} / 2+\alpha_{0}, & \text { if } & A_{* 0}<A<A_{* 1} \\
-U_{0 z}^{2}(0)\left(A-A_{* 0}\right)^{2} / 2+\alpha_{0}, & \text { if } & A \geqslant A_{* 1}
\end{array}\right\} .
$$

Again, for $\lambda=0$ there is only one extremum for $\Pi(A)$ and hence there can be no steady solutions. Again, this situation persists for small $\lambda>0$, but for sufficiently large $\lambda>0$ three extreme points emerge, and so again there will exist a family of periodic waves, which includes a solitary wave as a limiting case.

Let us consider an example of a stratified flow and show that solitary wave solutions and bore-like solutions are possible even if the nonlinearity in the final equation for the wave amplitude is only quadratic. We assume that the shear flow has the form

$$
U_{0}(z)-c=-0.135+0.7 z-0.075 z^{2}-0.4 z^{3} .
$$

Then we find that

$$
\begin{aligned}
& \Delta_{-}=-1.5 ; \Delta_{+}=1 ; U_{0 z}(0)=0.7 ; U_{0 z}(1)=-0.65 ; \gamma=0.017 ; \\
& A_{* 1}=1.538 ; A_{* 0}=2.143 .
\end{aligned}
$$

Since $\Delta_{-}<\Delta_{+} U_{0 z}(0) / U_{0 z}(1)$ and $A_{* 0}>A_{* 1}>0$ for this particular choice of parameters, we use (3.1) along with (3.2).

We look for solitary wave solutions of (3.1) and (3.2), which decay far upstream

$$
A \rightarrow 0, A_{X} \rightarrow 0, A_{X X} \rightarrow 0 \text { as } X \rightarrow-\infty,
$$

so that

$$
\alpha_{0}=-U_{0 z}^{2}(1) A_{* 1}^{2} / 2 .
$$




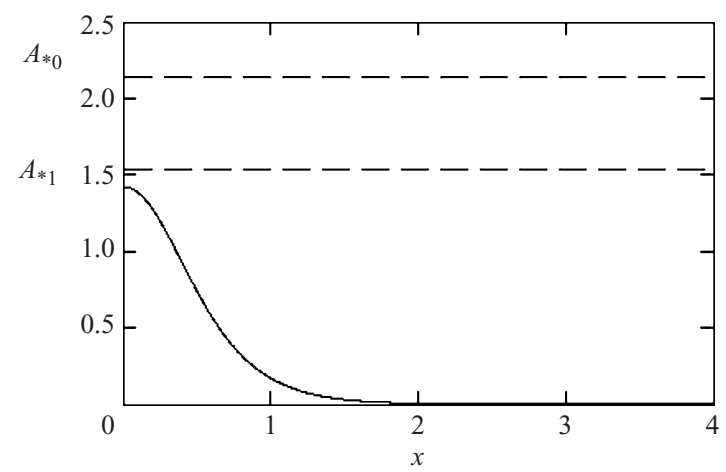

FIGURE 1. Profile of the amplitude function $A(x)$ for the case of a solitary wave. $\lambda / \mu^{2}=0.45$; $\mu=0.3$.

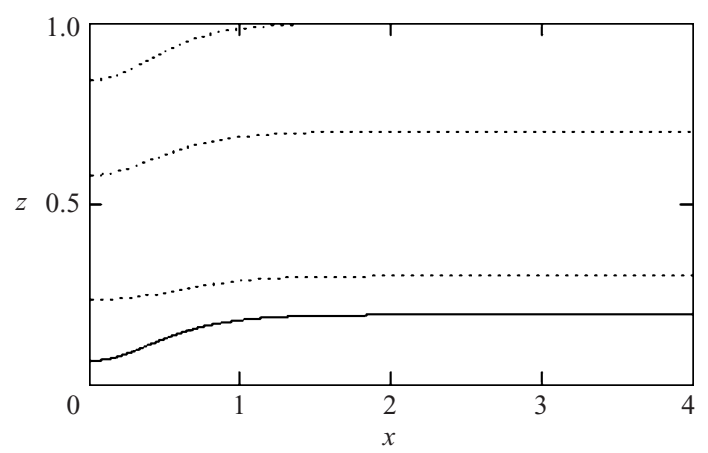

FIGURE 2. Streamline pattern (dotted lines) for the case of a solitary wave. Solid lines denote the boundaries of zones filled with mixed fluid. $\lambda / \mu^{2}=0.45 ; \mu=0.3$.

It can be readily shown that $A=0$ is an extremum of $\Pi(A)$ and a solitary wave solution for which $A \leqslant A_{* 1}$ for all $X$, has the amplitude

$$
A_{I \rightarrow I}=3 A_{* 1}-\frac{3 \lambda / \mu^{2}}{U_{0 z}^{2}(1)}, \quad \text { for } \frac{2 U_{0 z}^{2}(1) A_{* 1}}{3} \leqslant \frac{\lambda}{\mu^{2}} \leqslant U_{0 z}^{2}(1) A_{* 1} .
$$

An example of such a solitary wave solution satisfying (3.8) is shown in figures 1 and 2 . It is seen that a mixed zone exists only near the lower boundary in this case.

The inequalities in (3.8) define the interval of density stratification for which this solitary wave solution exists. The notation $A_{I \rightarrow I}$ indicates that the lower and the upper limits of the solitary wave solution belong to the zone $I$ of the amplitude range $\left(A \leqslant A_{* 1}\right)$. We denote the ranges $A_{* 1}<A<A_{* 0}$ and $A \geqslant A_{* 0}$ as zone $I I$ and zone $I I I$, respectively. For solitary waves with amplitudes within $A_{* 1}<A<A_{* 0}$ (zone $I I$ ) we find that

$$
A_{I \rightarrow I I}=\frac{\mu^{2}}{\lambda}\left[U_{0 z}^{2}(1) A_{* 1}^{2} / 2+\left\{\left(U_{0 z}^{2}(1) A_{* 1}^{2} / 2\right)^{2}-\frac{\lambda}{\mu^{2}} \frac{U_{0 z}^{2}(1) A_{* 1}^{3}}{3}\right\}^{1 / 2}\right],
$$

for $\left[U_{0 z}^{2}(1) A_{* 1}^{2}\left(A_{* 0}-A_{* 1} / 3\right)\right] / A_{* 0}^{2} \leqslant \lambda / \mu^{2} \leqslant 2 U_{0 z}^{2}(1) A_{* 1} / 3$. An example of a solitary wave solution satisfying (3.9) is shown in figures 3 and 4. According to (2.18), (2.24)(2.26), two mixed zones exist in this case. One is located near the lower boundary while another one appears near the upper boundary. 


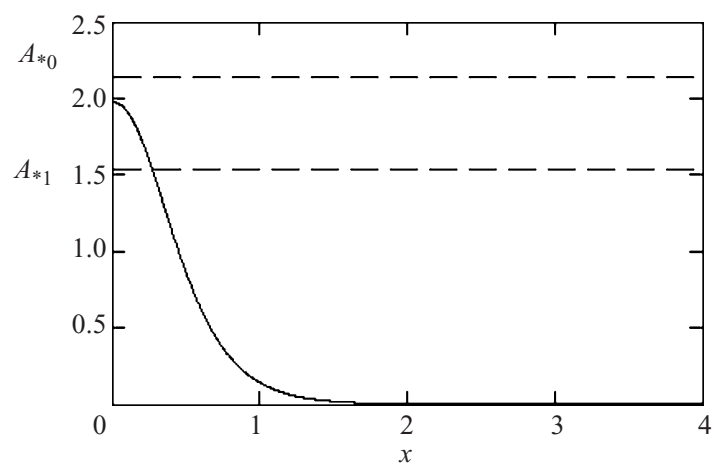

Figure 3. Profile of the amplitude $A(x)$ for the case of a solitary wave. $\lambda / \mu^{2}=0.375 ; \mu=0.3$.

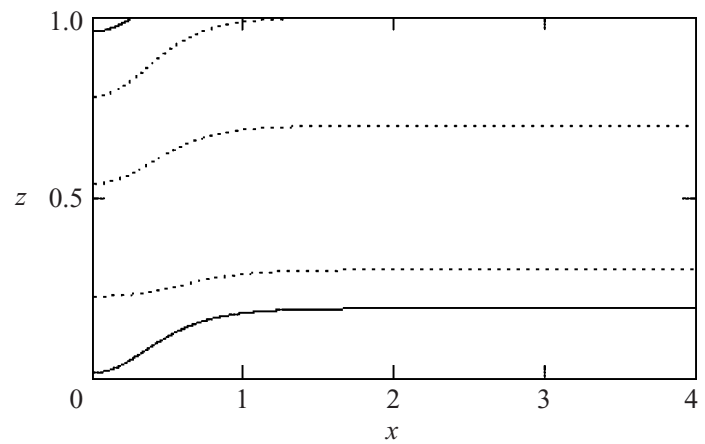

FIGURE 4. Streamline pattern (dotted lines) for the case of a solitary wave. Solid lines denote the boundaries of zones filled with mixed fluid. $\lambda / \mu^{2}=0.375 ; \mu=0.3$.

When $\lambda / \mu^{2}<\left[U_{0 z}^{2}(1) A_{* 1}^{2}\left(A_{* 0}-A_{* 1} / 3\right)\right] / A_{* 0}^{2}$, the amplitude of a solitary wave satisfying upstream conditions (3.6) along with the condition that this amplitude $A_{I \rightarrow I I I}>A_{* 0}$ can be found as the solution of a cubic equation $\Pi_{I I I}\left(A_{I \rightarrow I I I}\right)=\Pi_{I}(0)=0$, which is closest to $A=0$. The potential energy for the case $A>A_{*_{0}}$ can be easily defined from (3.2) and (3.7),

$$
\Pi_{I I I}(A)=\frac{\lambda}{\mu^{2}} \frac{A^{2}}{2}+\frac{U_{0 z}^{2}(1) A_{* 1}^{3}}{6}-\frac{U_{0 z}^{2}(1) A_{* 1}^{2} A}{2}-\frac{U_{0 z}^{2}(0)\left(A-A_{* 0}\right)^{3}}{2} .
$$

Physically the condition $\Pi_{I I I}\left(A_{I \rightarrow I I I}\right)=\Pi_{I}(0)=0$ means that the potential energy at the wave maximum and at far upstream are equal. The condition for a bore-like solution requires simultaneous satisfaction of (3.10) and so

$$
\left(\Pi_{I I I}\right)_{A}\left(A=A_{I \rightarrow I I I}\right)=0,
$$

that is, the amplitude $A=A_{I \rightarrow I I I}$ is also an extremum of the potential energy. This amplitude for $A>A_{* 0}$ is unique in the set of parameters chosen as a particular case in our study (3.5). Numerical solution of (3.5), (3.10) and (3.11) leads to $A_{I \rightarrow I I, \text { bore }}=3.54665$ at the specific stratification $\lambda / \mu^{2}=0.277108$. A plot of the internal bore is shown in figures 5 and 6 . Two semi-infinite mixed zones exist near the lower and the upper boundaries in accordance with (2.18), (2.24)-(2.26). The condition that three extrema of potential $\Pi$ exist to allow a stationary solution requires that 


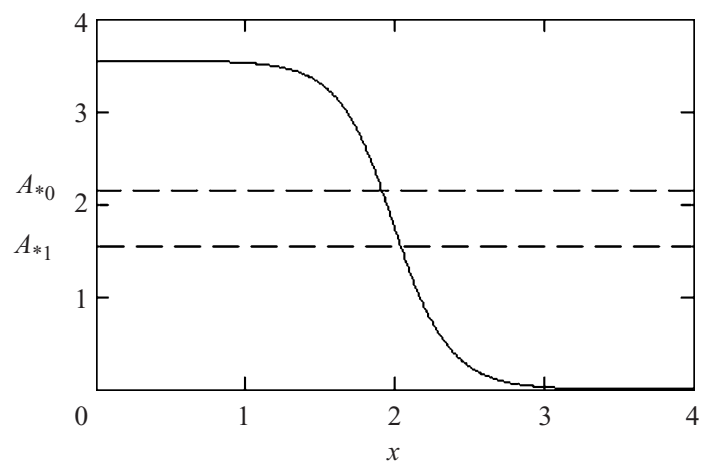

FIGURE 5. Profile of the amplitude $A(x)$ for the case of an internal bore $\lambda / \mu^{2}=0.277108$; $\mu=0.3$.

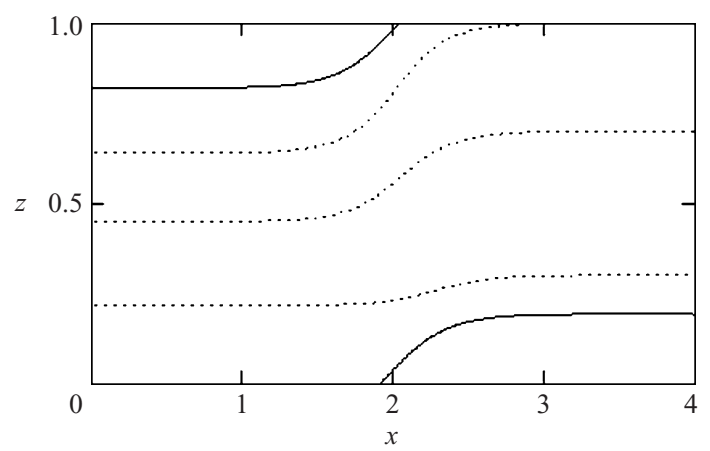

FIGURE 6. Streamline pattern (dotted lines) for the case of an internal bore. Solid lines denote the boundaries of zones filled with mixed fluid. $\lambda / \mu^{2}=0.277108 ; \mu=0.3$.

the minimum limiting strength of stratification is

$$
\frac{\lambda}{\mu^{2}}>U_{0 z}^{2}(0)\left(\sqrt{\frac{U_{0 z}^{2}(1) A_{* 1}^{2}}{U_{0 z}^{2}(0)}+A_{* 0}^{2}}-A_{* 0}\right) .
$$

It can be shown that the actual profile of the density stratification does not influence the solution, and the crucial parameter is the density difference (defined by $\lambda$, see (2.6)) across the flow confined between the critical layers. Other profiles of stratification can be examined similarly and lead to similar results.

\section{Conclusion}

In this paper we have studied vorticity waves propagating on an ambient shear flow, bounded by two critical layers which are adjacent to the rigid channel walls. Apart from this essential condition, the background shear flow can be quite arbitrary. In this case even small amplitude waves may overturn at a critical layer, followed by mixing and the subsequent formation of recirculation zones of a fluid with constant density. Our study allows for arbitrary, but weak ambient density stratification. The principal theoretical result of this study is a new evolution equation governing the amplitude of these vorticity waves. The quadratic nonlinear term accounts for both the effects of the ambient shear flow between the boundaries as well as the nonlinearity associated 
with the possible formation of recirculation zones due to the critical layers. However, due to these recirculation zones the nonlinear term can take a quite complicated form, and the coefficients may have either sign. This leads to unusual effects such as the formation of bore-like (front) steady solutions when the fluid is (weakly) density stratified. We have shown that there are no steady solutions in the case of a homogeneous fluid. We examined one particular case of a density-stratified shear flow and found the conditions which allow for the formation of steady solitary waves and bores. We have also shown that the existence of steady waves requires that the maximum wave amplitude is limited, which is quite different from the usual case of waves of small but finite amplitude when quadratic nonlinearity does not impose any restrictions on the wave amplitude.

\section{REFERENCES}

Benney, D. J. 1966 Long nonlinear waves in fluid flows. J. Math. Phys. 45, 52-63.

Derzho, O. \& Grimshaw, R. 1997 Solitary waves with a vortex core in a shallow layer of stratified fluid. Phys. Fluids 9, 3378-3385.

Derzho, O. \& Grimshaw, R. 2007 Asymmetric internal solitary waves with a trapped core in deep fluids. Phys. Fluids 19, 096601.

Grimshaw, R. 2001 Internal solitary waves. In Environmental Stratified Flows (ed. R. Grimshaw), Chapter 1, pp. 1-29. Kluwer.

Kachanov, Y. S., Ryzhov, O. S. \& SMith, F. T. 1993 Formation of solitons in transitional boundary layers: theory and experiments. J. Fluid Mech. 251, 273-297.

Maslowe, S. A. 1986 Critical layers in shear flows. Annu. Rev. Fluid Mech. 18, 405-432.

Maslowe, S. A. \& Redekopp, L. G. 1980 Long nonlinear waves in stratified shear flows. J. Fluid Mech. 101, 321-348.

Pedley, T. G. \& Stephanoff, K. D. 1985 Flow along a channel with a time-dependent indentation in one wall: the generation of vorticity waves. J. Fluid Mech. 160, 337-367.

SHRIRA, V. I. 1989 On the 'sub-surface' waves of the mixed layer of the upper ocean. Trans. USSR Acad. Sci., Earth Sci. Sec. 308, 276-279.

Voronovich, V. V., Shrira, V. I. \& Stepanyants, YU. A. 1998 Two-dimensional models for nonlinear vorticity waves in shear flows. Stud. Appl. Math. 100, 1-32. 